\title{
Effect of lateral masking and letter reversal on same-different judgments
}

\author{
LESTER E. KRUEGER and RALPH E. GOTT \\ Ohio State University, Columbus, Ohio
}

\begin{abstract}
Subjects judged whether two adjacent letters were identical or different under difficult conditions (brief, parafoveal, laterally masked letter pairs). Consistent with the noisy-operator theory, the increase in internal noise produced more spurious mismatches than matches and thus prompted greater rechecking of different pairs, because a large, nearly 70-msec speed advantage for same pairs was found, along with many false-different responses. Rechecking of spurious mismatches faltered (the fast-same effect remained the same or decreased, whereas false-different errors abounded), when particular letter pairs were presented mirror-reversed (Experiment 1) or had an outer (more peripheral) lateral mask (Experiment 2). Contrary to Wolford's featureperturbation model, backward masking greatly exceeded forward masking, especially with an outer mask.
\end{abstract}

According to the noisy-operator theory (Krueger, 1978), internal noise more often changes physical matches into perceived mismatches than vice versa, and, as a result, people recheck most perceived mismatches before responding "different." What happens if the level of internal noise is increased? Shepard and Metzler (1971) found a rather startling 1-sec fast-same effect with simultaneous presentation when they made the comparison more difficult by rotating one pattern relative to the other. Their subjects apparently rechecked perceived mismatches very carefully in order to catch spurious mismatches due to imperfect mental rotation or comparison.

However, an increase in spurious mismatches would increase the fast-same effect only if the criterion for rechecking were adjusted upward accordingly. Krueger (1985) found that subjects tended to use a common, compromise criterion when more difficult and less difficult letter pairs were intermixed rather than presented in separate blocks of trials. In the present study, the overall level of difficulty was consistently quite high, and therefore the general criterion ought to be adjusted upward accordingly, thus producing a much enhanced fast-same effect. Outer versus inner masking letter pairs were intermixed in both experiments, however, as were normal versus mirror-reversed target letter pairs in Experiment 1 , and it was expected that the more difficult pairs in these cases would show an increase in the preponderance of false-different responses, but not the fast-same effect (Egeth \& Blecker, 1971; Krueger, 1985).

\section{Feature-Perturbation Principle}

Presenting a masking letter pair on the more peripheral (outer) side of the target letter pair was expected to produce greater disruption (Mackworth, 1965). Accord-

Requests for reprints should be sent to Lester E. Krueger, Human Performance Center, Ohio State University, 404-B West 17th Avenue, Columbus, $\mathrm{OH} 43210$. ing to Wolford (1975), this is because feature movement during letter identification tends to occur in the direction of the fovea. If so, then a feature-spewing lateral mask, especially an outer mask, ought to be most disruptive when presented simultaneously with or immediately preceding the target (forward masking).

Krumhansl (1977) found greater forward than backward masking between two letters presented sequentially for 75 msec each. With simultaneous presentation, she obtained asymmetrical lateral interference, but with sequential presentation, she found that relative position (inner, outer) mattered only for the second letter, which indicates that "feature perturbations from the first letter may affect identification of the second letter"' (p. 300), but not vice versa. However, she filled each letter position with a noise character before and after its presentation, and it may be that the returning noise characters interrupted processing to a greater extent on the second letter. When Chastain (1982) omitted the noise characters, he found asymmetrical lateral interference only with simultaneous presentation, and he generally found greater backward than forward masking.

Krumhansl (1977) and Chastain (1982) used only three levels of SOA $(-75,0,+75 \mathrm{msec}$; a negative value indicates forward masking). In Experiments 1 and 2 here, a greater variety and range of SOA values were used in order to determine more precisely the point of peak interference. In Experiment 1, target pairs were presented in all four cardinal directions (above, below, left, right), rather than just left and right, as in Experiment 2, in order to test whether the inner-outer asymmetry depends on horizontal, left-to-right scanning.

\section{EXPERIMENT 1}

\section{Method}

Subjects. Thirty undergraduates participated. All had at least 20/30 (corrected) vision. Data were excluded on eight additional subjects, whose errors exceeded $25 \%$. 
Apparatus and Stimuli. Uppercase letters were presented at $32-\mathrm{cd} / \mathrm{m}^{2}$ intensity on a P31 phosphor by an Imlac PDS-4 graphics computer. Target letters were selected randomly from the set F, G, J, $\mathrm{K}, \mathrm{L}$. The masking letter was always $\mathrm{O}$. Each letter was $.29 \mathrm{~cm}\left(.24^{\circ}\right)$ wide and $.43 \mathrm{~cm}\left(.35^{\circ}\right)$ high, and the two letters in a pair were spaced $.19 \mathrm{~cm}\left(.16^{\circ}\right)$ apart vertically (left, right visual fields) or horizontally (upper, lower visual fields). A target letter and its adjoining masking letter were separated by one letter width $(.29 \mathrm{~cm})$. All four inner or outer masking pairs (left, right, upper, lower) were shown in order not to disclose the location of the target pair. The target pair, inner masking pair, and outer masking pair were located $2.51,1.93$, and $3.09 \mathrm{~cm}$ $\left(2.05^{\circ}, 1.58^{\circ}\right.$, and $\left.2.53^{\circ}\right)$, respectively, from the fixation mark.

The 480 regular trials (preceded by 40 practice trials) represented all combinations of the following factors: (1) SOA, (2) letter orientation of target pair (normal, left-right mirror-reversed), (3) trial type (same, different), (4) visual field of target pair (left, right, upper, lower), (5) relative position of masking pair (inner, outer) $(15 \times 2 \times 2 \times 4$ $\times 2=480$ ).

Procedure. When the subject pressed a footpedal, the target pair appeared $800 \mathrm{msec}$ later for $100 \mathrm{msec}$. The delay for the masking array, which was presented for $17 \mathrm{msec}$, varied from $500 \mathrm{msec}(-300$-msec SOA) to $1,100 \mathrm{msec}(+300$-msec SOA).

The subjects were instructed to ignore the masking array, to keep their eyes focused on the fixation mark (a small plus sign), to attend only to the target pair, and to respond as quickly as possible, but not at the expense of accuracy. Feedback on response accuracy appeared for $2 \mathrm{sec}$ in the upper left part of the screen, which may have led subjects to favor that side, so only the effect of horizontal (left, right) versus vertical (upper, lower) visual field will be reported.

Half of the subjects pressed a right-hand button for same judgments and a left-hand button for different judgments, and the other half had the reverse hand assignment. Trials having a response time (RT) above $3 \mathrm{sec}$ or below $200 \mathrm{msec}$ were discarded.

\section{Results}

Owing to missing cells on correct RTs, the first analysis collapsed the SOAs into early $(-300$ to $-100 \mathrm{msec})$,

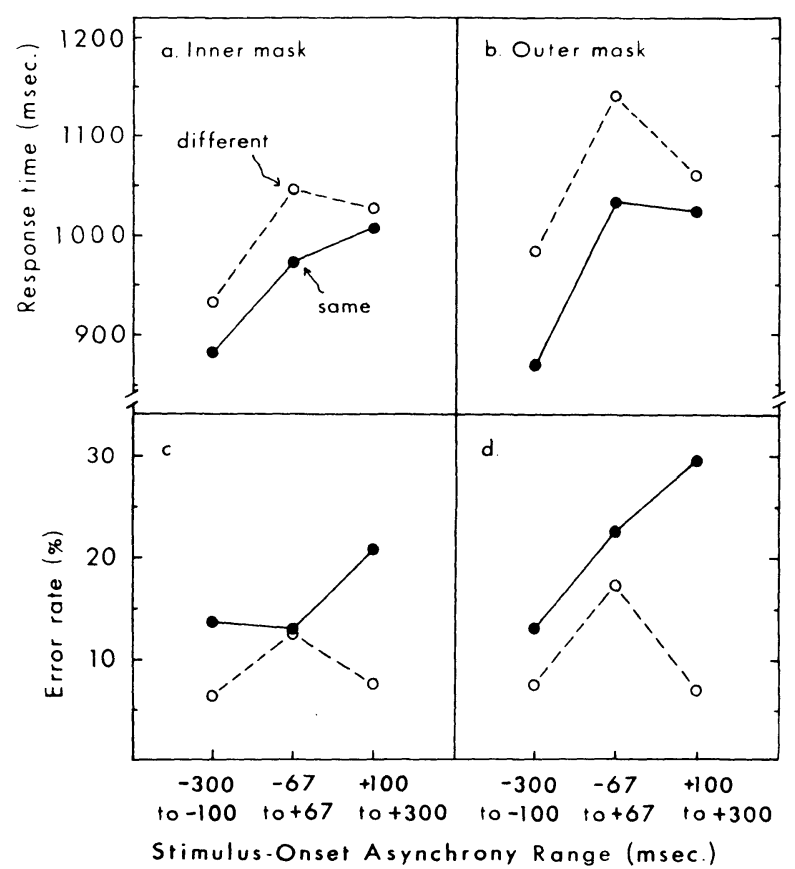

Figure 1. Experiment 1: Mean RT and percentage of error rate by trial type, target-mask SOA range, and relative mask position.

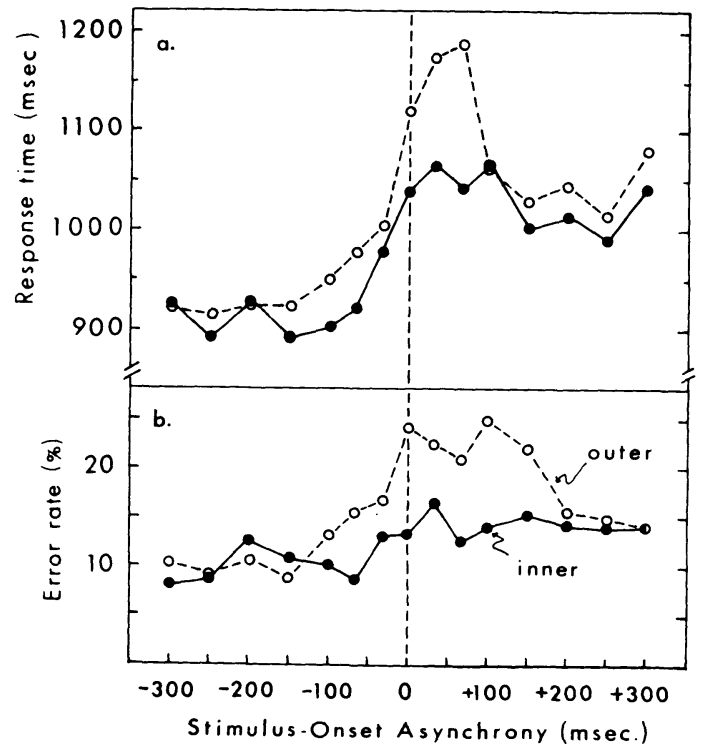

Figure 2. Experiment 1: Mean RT and percentage of error rate by target-mask SOA and relative mask position.

middle or nearly simultaneous ( -67 to $+67 \mathrm{msec}$ ), and late $(+100$ to $+300 \mathrm{msec}$ ) masking ranges (see Figure 1). Mean RTs were lower on same (vs. different) pairs $[F(1,28)=14.11, p<.001]$ and on normal (vs. mirrorreversed) pairs $[\mathrm{F}(1,28)=11.60, \mathrm{p}<.01]$. Errors were higher on same pairs $[\mathrm{F}(1,28)=45.49, \mathrm{p}<.001]$ and on mirror-reversed pairs $[\mathrm{F}(1,28)=4.81, \mathrm{p}<.05]$. Letter orientation interacted with trial type (same, different) on errors $[F(1,28)=4.32, p<.05]$ but not on $R T$ $[F(1,28)=1.97]$; letter reversal increased errors by $2.77 \%$ and $0.04 \%$ on same and different trials, respectively, and RT by 31 and $12 \mathrm{msec}$. Letter reversal increased RT more when the mask was outer rather than inner $(37$ vs. $7 \mathrm{msec})[\mathrm{F}(1,28)=4.88, \mathrm{p}<.05]$. Trial type interacted with relative mask position (inner, outer) on both RT $[\mathrm{F}(1,28)=6.49, \mathrm{p}<.025]$ and errors $[\mathrm{F}(1,28)=8.39, \mathrm{p}<.01]$. The tendency for same trials to be faster but less accurate was much stronger with an outer mask. Trial type interacted with SOA range on both $\operatorname{RT}[\mathrm{F}(2,56)=7.88, \mathrm{p}<.001]$ and errors $[\mathrm{F}(2,56)=$ $28.92, \mathrm{p}<.001]$. A nearly simultaneous mask increased the speed advantage for same trials and decreased the predominance of false-different errors, whereas a late mask, which apparently terminated rechecking prematurely, did just the opposite.

The second analysis collapsed the data across trial type and letter orientation. As shown in Figure 2, judgments were slower $[F(1,28)=21.02, p<.001]$ and less accurate $[\mathrm{F}(1,28)=38.95, \mathrm{p}<.001]$ with an outer (vs. inner) masking array. The SOA also had a significant effect on both RT $[F(14,392)=26.04, p<.001]$ and errors $[F(14,392)=10.34, p<.001]$, with positive SOAs (backward masking) producing greater lateral interference. 
Relative mask position interacted with SOA on both RT $[F(14,392)=3.54, p<.001]$ and errors $[F(14,392)=$ $3.92, \mathrm{p}<.001]$. The greater effect of the outer mask was especially evident with backward masking, both on RT (SOA of 0 to $+67 \mathrm{msec}$ ) and errors (SOA of 0 to $+150 \mathrm{msec}$ ). Relative mask position did not interact significantly with visual field, and in separate analyses, RT and errors were significantly greater with the outer (vs. inner) mask, both on the vertical axis (upper, lower pairs) and on the horizontal axis (left, right pairs) $(\mathrm{p}<.01$ or better in all cases).

\section{Discussion}

The great preponderance of false-different errors obtained here indicates that internal noise produced many spurious mismatches. The 67msec fast-same effect far exceeds the typical 20 -msec effect obtained with simultaneous presentation under normal conditions. Having trials that were consistently quite difficult apparently permitted considerable criterion adjustment, as Krueger (1985) found.

Rechecking faltered in one case, however, in which trials were intermixed. An unfamiliar letter orientation reduced (insignificantly) the fastsame effect and increased the preponderance of false-different errors. Inexplicably, there was no sign of any faltering in rechecking on outer masking pairs, which were intermixed with inner masking pairs and yet produced an enhanced fast-same effect.

Backward masking exceeded forward masking, especially with an outer mask. Lateral interference generally peaks at an SOA of +50 to +100 msec (Kahneman, 1968), which was the case here, especially on RT (see Figure 2). If Wolford's (1975) perturbation model were correct, then forward masking ought to have predominated, especially with an outer mask.

\section{EXPERIMENT 2}

In Experiment 2, the target-mask distance was varied in order to vary the task difficulty and to provide a more sensitive test of Wolford's (1975) perturbation model.

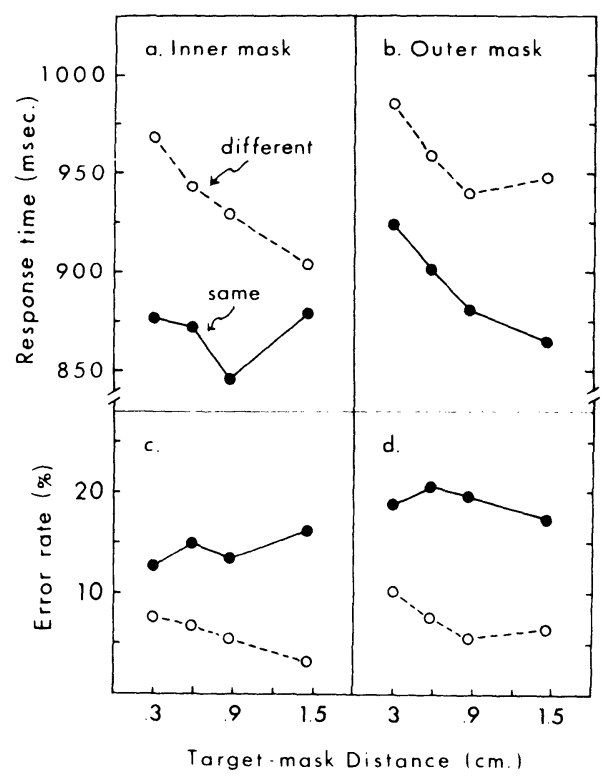

Figure 3. Experiment 2: Mean RT and percentage of error rate by trial type, target-mask distance, and relative mask position.
Mainly positive SOAs were used, since backward masking had predominated in Experiment 1.

\section{Method}

There were 60 subjects. Data were excluded on 16 additional subjects whose errors exceeded $25 \%$.

The method was the same as that in Experiment 1, except for the following changes. Only normally oriented letter pairs located in the left or right visual fields were used. The target-mask distance was $.29, .58$, .87 , or $1.45 \mathrm{~cm}\left(.24^{\circ}, .47^{\circ}, .71^{\circ}\right.$, or $\left.1.18^{\circ}\right)$. The most central inner mask was $.77 \mathrm{~cm}\left(.63^{\circ}\right)$ from the fixation mark, whereas the most peripheral outer mask was $4.25 \mathrm{~cm}\left(3.47^{\circ}\right)$ from the fixation mark.

The 320 regular trials (preceded by 30 practice trials) represented all combinations of the following factors: (1) SOA, (2) trial type (same, different), (3) visual field of target pair (left, right), (4) relative position of masking pair (inner, outer), and (5) target-mask distance (.29, $.58, .87,1.45 \mathrm{~cm})(10 \times 2 \times 2 \times 2 \times 4=320)$. Each trial began immediately following the preceding trial's 2 -sec feedback message, which was centered above the fixation mark.

\section{Results}

The first analysis collapsed the data across levels of SOA. Same trials were faster $[\mathrm{F}(1,58)=44.30$, $\mathrm{p}<.001]$ and more errorful $[\mathrm{F}(1,58)=122.70$, $\mathrm{p}<.001]$. The preponderance of false-different errors was enhanced with an outer (vs. inner) mask (11.67\% vs. $8.54 \%)[\mathrm{F}(1,58)=6.21, \mathrm{p}<.025]$, but the fast-same effect was not $(65$ vs. $67 \mathrm{msec})(\mathrm{F}<1)$, which indicates that rechecking faltered. Trial type interacted with targetmask distance on errors $[\mathrm{F}(3,174)=7.13, \mathrm{p}<.001]$. As distance decreased, only false-same responses steadily increased (see Figure 3 ). Distance significantly affected errors on different trials analyzed separately $[\mathrm{F}(3,174)=$ $9.72, \mathrm{p}<.001]$ but not on same trials $[\mathrm{F}(3,174)=1.35]$.

The left and right visual fields did not differ on either RT or errors ( $\mathrm{F} \leq 1.46)$, and the fast-same effect did not differ between the two fields (60 vs. $73 \mathrm{msec}$ ) (F< 1). However, the inner-outer asymmetry was larger in the left (vs. right) field, both on RT (40 vs. $7 \mathrm{msec}$ ) $[\mathrm{F}(1,58)=8.66, \mathrm{p}<.01]$ and errors $(4.23 \%$ vs. $2.31 \%)$ $[\mathrm{F}(1,58)=5.22, \mathrm{p}<.05]$.

The second analysis collapsed the data across trial type and visual field on RT (Figure 4) and errors (Figure 5). An outer (vs. inner) mask increased both RT $[F(1,58)=$ $9.79, \mathrm{p}<.01]$ and errors $[\mathrm{F}(1,58)=34.30, \mathrm{p}<.001]$. Greater lateral interference occurred at positive SOAs (backward masking), both on $\mathrm{RT}[\mathrm{F}(9,522)=4.71$, $p<.001]$ and errors $[F(9,522)=2.33, p<.025]$. Increasing the target-mask distance reduced both RT $[F(3,174)=16.15, \mathrm{p}<.001]$ and errors $[\mathrm{F}(3,174)=$ $2.84, \mathrm{p}<.05]$.

The effect of SOA was greater with an outer (vs. inner) mask, both on $\operatorname{RT}[F(9,522)=2.23, \mathrm{p}<.025]$ and errors $[\mathrm{F}(9,522)=5.41, \mathrm{p}<.001]$, and it also increased as the target-mask distance decreased, both on RT $[\mathrm{F}(27,1566)=1.49, \mathrm{p}<.05]$, and, although not quite significantly so, on errors $[F(27,1566)=1.44, p<.10]$. It was especially large on errors when the mask was both outer and spatially close $[\mathrm{F}(27,1566)=1.80, \mathrm{p}<.01]$. Relative mask position (inner, outer) did not interact with target-mask distance (F $\leq 1.18$ on both RT and errors). 


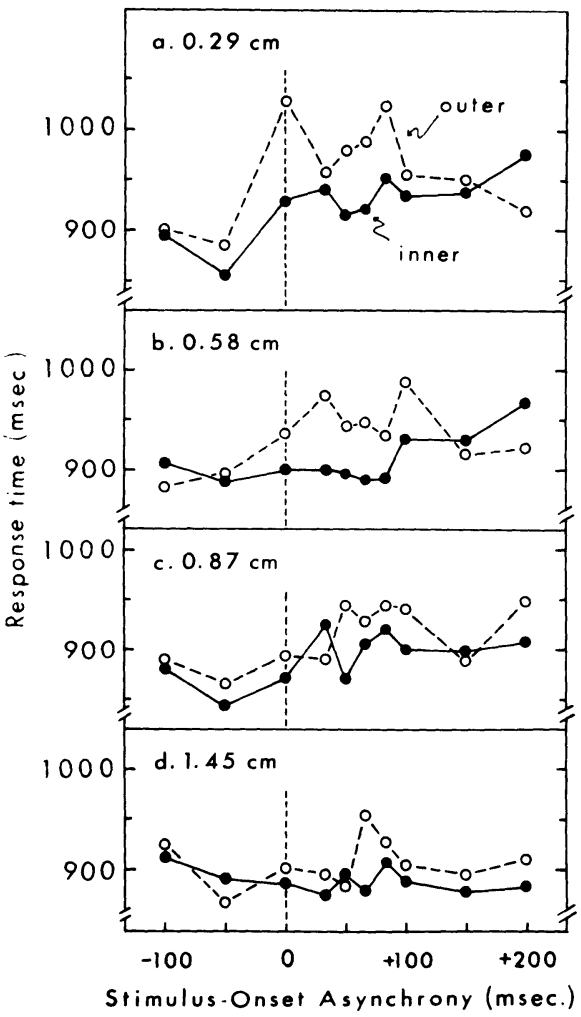

Figure 4. Experiment 2: Mean RT by target-mask SOA, targetmask distance, and relative mask position.

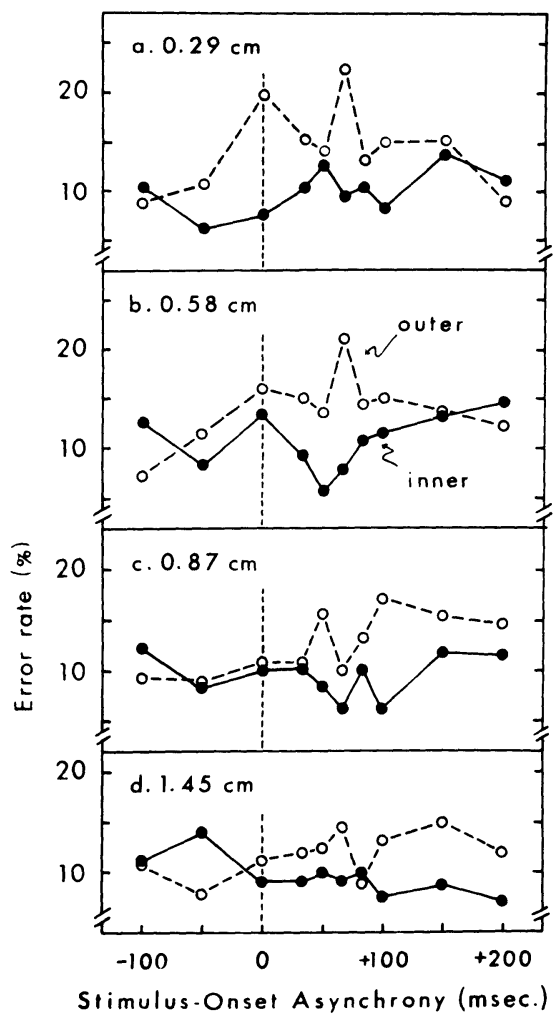

Figure 5. Experiment 2: Percentage of error rate by target-mask SOA, target-mask distance, and relative mask position.
In separate analyses at each distance, the outer mask consistently produced greater errors $(\mathrm{p}<.025$ or better in all cases) and produced greater RTs at $.29 \mathrm{~cm}(\mathrm{p}<.01)$ and $.87 \mathrm{~cm}(\mathrm{p}<.05)$.

\section{Discussion}

In Experiment 2, moving the masking pair toward the target pair seemingly biased the judgment toward same. Apparently, the "same" signal evoked by the matching features in the symmetrical masking pair (two Os) tended more and more to supplement that of the target pair as the two approached (Eriksen, O'Hara, \& Eriksen, 1982). An outer (vs. inner) mask mainly increased the false-different errors, however, and therefore it produced greater disruption of, not grouping with, the target pair (cf. Banks, Bachrach, \& Larson, 1977; Banks, Larson, \& Prinzmetal, 1979).

Experiment 2, like Experiment 1, obtained no evidence of feature perturbations. Forward masking did not exceed backward masking, and the predominance of backward masking was not any weaker with the outer mask. If mask features tend to migrate inward, as Wolford (1975) claimed, lateral interference from an outer mask ought to peak ever earlier (lower SOA value) as the target-mask distance is increased, but Figures 4 and 5 show just the opposite tendency.

In Experiment 1, the inner-outer asymmetry was evident on the vertical axis, which indicates a sensory locus that does not involve horizontal scanning (Chastain, 1982). In Experiment 2, the inner-outer asymmetry was greater in the left (vs. right) field, which indicates an additional higher level locus that does involve horizontal scanning. The present results thus support the two-stage models of Schissler and Barata (1972) and White (1976), in which peripheral-to-foveal scanning occurs early in iconic processing and is followed by left-to-right scanning.

\section{REFERENCES}

Banks, W. P., Bachrach, K. M., \& Larson, D. W. (1977). The asymmetry of lateral interference in visual letter identification. Perception \& Psychophysics, 22, 232-240.

Banks, W. P., Larson, D. W., \& Prinzmetal, W. (1979). Asymmetry of visual interference. Perception \& Psychophysics, 25, 447-456.

Chastain, G. (1982). Masking and the identification of characters presented simultaneously and sequentially to the parafovea. Psychological Research, 44, 135-145.

EGETH, H., \& BLECKER, D. (1971). Differential effects of familiarity on judgments of sameness and difference. Perception \& Psychophysics, 9, $321-326$.

Eriksen, C. W., O'Hara, W. P., \& Eriksen, B. (1982). Response competition effects in same-different judgments. Perception \& Psychophysics, 32, 261-270.

KAHNEMAN, D. (1968). Method, findings, and theory in studies of backward masking. Psychological Bulletin, 70, 404-425.

Krueger, L. E. (1978). A theory of perceptual matching. Psychological Review, 85, 278-304.

KRUEGER, L. E. (1985). Effect of intermixed foveal and parafoveal presentation on same-different judgments: Evidence for a criterioninertia model. Perception \& Psychophysics, 37, 266-271.

KRUMHANSL, C. L. (1977). Naming and locating simultaneously and sequentially presented letters. Perception \& Psychophysics, 22, 293-302.

Mackworth, N. H. (1965). Visual noise causes tunnel vision. Psychonomic Science, 3, 67-68.

SCHISSLER, D., \& BARATA, M. (1972). Evidence for an addition to the reading scan hypothesis. Perception \& Psychophysics, 12, 221-223.

ShePARD, R. N., \& METZleR, J. (1971). Mental rotation of threedimensional objects. Science, 171, 701-703.

WhITE, M. J. (1976). Order of processing in visual perception. Canadian Journal of Psychology, 30, 140-156.

Wolford, G. (1975). Perturbation model for letter identification. Psychological Review, 82, 184-199.

(Manuscript received August 15, 1984; revision received for publication June 11, 1985.) 